\title{
Alzheimer's Pathogenesis and Its Link to the Mitochondrion
}

\author{
C. Simoncini, D. Orsucci, E. Caldarazzo Ienco, G. Siciliano, U. Bonuccelli, and M. Mancuso \\ Department of Experimental and Clinical Medicine, Neurological Clinic, University of Pisa, Via Roma 67, 56126 Pisa, Italy \\ Correspondence should be addressed to M. Mancuso; mancusomichelangelo@gmail.com
}

Received 30 December 2014; Revised 16 March 2015; Accepted 2 April 2015

Academic Editor: Trevor A. Mori

Copyright (C) 2015 C. Simoncini et al. This is an open access article distributed under the Creative Commons Attribution License, which permits unrestricted use, distribution, and reproduction in any medium, provided the original work is properly cited.

\begin{abstract}
Alzheimer's disease (AD) is the most common form of dementia in the elderly. This neurodegenerative disorder is clinically characterized by impairment of cognitive functions and changes in behaviour and personality. The pathogenesis of AD is still unclear. Recent evidence supports some role of mitochondria dysfunction and oxidative stress in the development of the neurodegenerative process. In this review, we discuss the role of mitochondrial dysfunction in $\mathrm{AD}$, focusing on the mechanisms that lead to mitochondrial impairment, oxidative stress, and neurodegeneration, a "vicious circle" that ends in dementia.
\end{abstract}

\section{Alzheimer's Disease and Mild Cognitive Impairment}

Alzheimer's disease (AD) is a degenerative brain disorder separately described in 1906 and 1910 by Alzheimer and Perusini [1-3]. AD is the most common form of dementia, affecting over 24 million of people worldwide, with 4.6 million new cases per year [4]. Usually, at the time of onset, age is between 60 and 70 years. The disease is clinically characterized by a chronic and disabling memory impairment in association with at least one other cognitive feature, resulting in a reduced competence to judge or to reflect. Cognitive functions that can be involved include the verbal and nonverbal memory, language, orientation, constructional abilities, abstract thinking, ability to solve problems, or praxis. There are frequently changes in behaviour and personality. Usually, death is caused by infections, pulmonary embolism, malnutrition, or heart disease. A definite diagnosis is only possible postmortem with autopsy [5]. The most common pathological features of $\mathrm{AD}$ include selective pyramidal neuronal death, accumulation of intraneuronal and extracellular fibrils, neurofibrillary tangles (NFT), and senile plaques [6, 7]. Senile plaques are filamentous aggregates of amyloid-beta peptide $(\mathrm{A} \beta)$ [8], whereas the main component of neurofibrillary tangles is the microtubule-associated protein tau [9]. These pathological deposits can be found during autopsy. In vivo, these aggregates may be detected among $\mathrm{AD}$ patients by imaging techniques such as positron emission tomography
(PET) with Pittsburg compound B for amyloid [10] or 18FTHK23 for Tau aggregates [11], although further clinical studies are still needed to define the correct assay.

Mild cognitive impairment (MCI) is an intermediate stage between normal cognitive function and dementia [12], with deficits in at least one cognitive domain [13]. There are two forms of MCI: the amnestic MCI (memory impairment) and the nonamnestic MCI (multiple-MCI) with impairment in cognitive domains other than memory (e.g., frontal/ executive, language, attention, or visuospatial skills) [14].

Currently, the pathogenesis of AD and the mechanisms leading to neuronal death have not been fully clarified. AD may be familial (FAD) and sporadic (SAD).

FAD represents a minority of $\mathrm{AD}$ cases, with autosomal dominant inheritance. Mutations in three known genes can cause FAD. These genes encode for amyloid precursor protein (APP) and presenilin-1 and presenilin-2 (PS1 and PS2) [15]. To date, the beta-amyloid cascade hypothesis remains the main pathogenetic model of FAD [16]. The $\mathrm{A} \beta$ peptide is the result of a regulated intramembrane proteolysis of APP by the sequential cleavage by $\beta$ - and $\gamma$-secretases $[17,18]$. A $\beta$ plaques may be the cause of neurotoxicity, loss of synapses, and, ultimately, neuronal death $[19,20]$. The exact mechanisms of the neurotoxicity of $\mathrm{A} \beta$ are still unknown. Several evidence suggest that $\mathrm{A} \beta$ exerts its toxicity intracellularly [21, 22]. Neurotoxicity of amyloid might play an important role in cell death by a cascade of events resulting in reactive oxygen species generation and oxidative stress [23]. Since 
mitochondria are the main site of free radical production [24], their involvement in neuronal degeneration has been hypothesized.

The main risk factor for SAD is aging [25]. Probably, SAD is the result of a complex interplay between acquired and genetic factors [26].

\section{Positron Emission Tomography and Energy Metabolism in AD}

In $\mathrm{AAD}$, the cerebral metabolic rate for glucose is reduced in temporoparietal cortices; fluorodeoxyglucose positron emission tomography (FDG PET) can measure this reduction. This feature appears to relate to the clinical disabilities of $\mathrm{AD}$ patients and can also precede the clinical symptoms by decades [27]. Reduced cerebral metabolism preceded both the neuropsychological impairment and the cortical atrophy detected with conventional neuroimaging [28].

In agreement with these studies, decreased glucose utilization in skin fibroblasts of $\mathrm{AD}$ patients has been also observed [29]. Mitochondria are membrane-enclosed cytoplasmatic organelles, defined as "the powerhouse of all cells"; mitochondrial dysfunction will inevitably impair energy metabolism. In brain, the oxidation of glucose occurs through the tricarboxylic acid (TCA) cycle (the Krebs' cycle) in the mitochondria. Acetyl CoA, the metabolite necessary to initiate the TCA cycle, is obtained by the oxidative decarboxylation of pyruvate, the product of glycolysis, through the pyruvate dehydrogenase complex (PDHC), which includes eight different enzymes. Deficiency in the two key enzymes of rate limiting step of the TCA cycle, PDHC and $\alpha$-ketoglutarate dehydrogenase complex (KGDHC), can explain the observed defects in glucose metabolism in the $\mathrm{AD}$ brains $[30,31]$. In addition to reduced rate of glucose, other alterations in $\mathrm{AD}$ brains include decreased activity of isocitrate dehydrogenase and increased activity of succinate dehydrogenase and malate dehydrogenase [31]. All the changes observed in TCA cycle activities correlated with clinical state, suggesting that they could lead to diminished brain metabolism resulting in the decline in brain function [31].

Liang and coworkers, in a recent transcriptomic study, examined the expression of 80 metabolically relevant nuclear genes in brains of $\mathrm{AD}$ patients, comparing them to normal controls [32]. This study showed a significantly lower expression of the nuclear genes encoding subunits of the mitochondrial electron transport chain (ETC) in posterior cingulate cortex, in the middle temporal gyrus, in hippocampal CA1 cells, in entorhinal cortex, and in other brain areas of $\mathrm{AD}$ patients [32]. Western blots confirmed underexpression of the ETC subunits [32]. Thus, metabolic abnormalities in FDG PET studies of $\mathrm{AD}$ are associated with reduced neuronal expression of nuclear genes encoding subunits of the mitochondrial ETC.

\section{Mitochondria and the Mitochondrial DNA}

Mitochondria range from 0.5 to 10 micrometers $(\mu \mathrm{m})$ in diameter and have developed from a symbiotic relationship established 1.5 billion years ago between primitive aerobic bacteria with a single-cell anaerobic organism [33]. Mitochondria are ubiquitous in eukaryotes. They are composed of a smooth outer membrane surrounding an inner membrane of significantly larger surface area that, in turn, surrounds a protein-rich core, the matrix. They contain 2 to 10 molecules of DNA, the mitochondrial DNA (mtDNA) [34]. Their number per cell ranges from zero in erythrocytes to several thousands in striated muscle cells. Their main function is to support aerobic respiration and to provide energy as adenosine triphosphate (ATP), by means of the electron transport chain (ETC), which is needed for oxidative phosphorylation that provides the most efficient energetic outcome in terms of ATP production for cell. The ETC consists of four multimeric protein complexes (complexes I to IV) located in the inner mitochondrial membrane together with complex V [34]. The ETC also requires cytochrome c (cyt c) and a small electron carrier, coenzyme Q10 (CoQ10 or ubiquinone). Electrons are transported along the complexes to molecular oxygen $\left(\mathrm{O}_{2}\right)$, finally producing water. At the same time, protons are pumped across the mitochondrial inner membrane, from the matrix to the intermembrane space, by the complexes I, III, and IV. This process creates an electrochemical proton gradient. ATP is produced by the influx of these protons back through the complex V, or ATP synthase (the "rotary motor") [35].

Mitochondria are key regulators of cell survival and death. Dysfunction of mitochondrial energy metabolism leads to reduced ATP production, impaired calcium buffering, and increased generation of reactive oxygen species (ROS) [36]. Human mtDNA is a circular, double-stranded molecule of 16569 base pairs. It contains 37 genes, including 13 protein-encoding genes, 22 transfer RNA genes, and two ribosomal RNA genes. All 13 protein-encoding genes are components of the mitochondrial respiratory chain, which is located in the inner membrane [34].

\section{Mitochondria and Oxidative Stress}

Oxidative stress describes the condition in which cellular antioxidant defences are insufficient to keep ROS levels below a toxic threshold [37]. The accumulation of ROS damages several biomolecules, including lipids, proteins, and nucleic acids. The cells possess a complex network of defence mechanisms to neutralize excessive accumulation of ROS. Some tissues, especially the brain, are mostly vulnerable to oxidative stress because of their elevated consumption of oxygen and the consequent generation of large amounts of ROS. Moreover, compared to other tissues, the brain, from one hand, has a lower activity of antioxidant enzymes such as glutathione peroxidase and catalase and, to the other hand, it contains elevated concentrations of polyunsaturated fatty acids that are highly susceptible to lipid peroxidation [38]. Mitochondria generate ATP as electrons flow through mitochondrial complexes I to IV of the electron transport chain, from donors with lower redox potential to acceptors with higher redox potential [39]. The final acceptor is oxygen that is reduced to water. The generated energy drives the phosphorylation of ADP to ATP by the mitochondrial 
complex V (or ATP-synthase). Although, the transport of the electrons through mitochondrial complexes is an efficient process, ROS may be produced and this amount is higher in damaged and aged mitochondria. In several neurodegenerative diseases, mitochondrial dysfunction is one of the earliest and prominent features [40-42], leading to increased oxidative stress neurons [43]. Additionally, mitochondria are a target for ROS, causing the oxidation of their components such as mtDNA, lipids, and proteins in a vicious circle [44].

\section{Mitochondrial Oxidative Stress and AD}

The possible relationship between oxidative stress and $\mathrm{AD}$ originally derived from the free radical hypothesis of aging, suggesting that age-related accumulation of ROS could be responsible of damage to major cell components [36]. ROS increase is consequent to mitochondrial dysfunction linked to aging and mitochondrial dysfunction results to be accelerated in $\mathrm{AD}$ patients [45]. As demonstrated by several studies, mitochondrial dysfunction and increased oxygen species generation play an important role in $\mathrm{AD}$ $[25,36]$. Increased oxygen species production and deficient mitochondrial dynamic balance have been suggested to be both reason and consequence of $\mathrm{AD}$ related pathology. This event may correlate with the presence of dysfunctional mitochondria, resulting in reactive oxygen species generation (ROS) [46]. Mitochondria generate cell energy as electrons flow through electron transport chain. Although this mechanism is an efficient process, some ROS may be produced and dysfunctional mitochondria can generate high levels of ROS with a toxic effect for cells with a long life span and a deficiency in antioxidant defences, including cerebral cells [43]. Furthermore, mitochondria may be themselves damaged by ROS, causing a "vicious circle," resulting in additional mitochondrial impairment. Aging-related oxidative damage may be a significant risk factor for $\mathrm{AD}$ [25]. CNS functions heavily depend on efficient mitochondrial activity, since brain tissue requires high energy provision. However, it is unclear whether mitochondrial impairment and oxidative stress are actually involved at the onset or in the progression of the neurodegenerative process.

Trials with antioxidant agents such as vitamin $\mathrm{E}$ have shown controversial results; Sano and coworkers [47] demonstrated that vitamin $\mathrm{E}$ slows the progression of disease in moderately severe $\mathrm{AD}$ while Petersen et al. did not demonstrated a significant benefit of this antioxidant agent in patients with MCI [48]. Dumont and colleagues examined the role of Coenzyme Q10 in the Tg19959 transgenic mouse model of $\mathrm{AD}$ that overexpress $\mathrm{A} \beta \mathrm{PP}$, demonstrating a positive effect of this factor both on pathology and behaviour in this model [49].

\section{6. “Cybrid” Models of AD}

Cybrid cells are generated by mixing the contents of nonnucleated cells or cytoplasts, with nucleated cells [50]. A particular case of cybrid cells is formed by the fusion of cells completely private of mitochondrial DNA and containing only nuclear DNA (nDNA), the so-called cell rho-zero, with cytoplasts containing only mitochondrial DNA (mtDNA). It is important to note that cybrid cell lines are not created through the transfer of isolated mtDNA, but rather through the transfer of whole mitochondria to $\rho 0$ cells. All transferred cytoplast components that cannot be perpetuated independently of the host cell nucleus degrade over time and dilute over the course of repeated cell divisions. Therefore, any transferred cytoplast component that cannot perpetuate itself independent of the cell nucleus should not have a sustainable molecular or biochemical effect. Theoretically, the only selfperpetuating component is the mtDNA. These cybrid cells can be a model to study central nervous disease, such as AD. The first studies of cybrid cell lines containing mtDNA from AD patients were published in 1997 [50]. These studies demonstrated that, in $\mathrm{AD}$ cybrids, cytochrome $\mathrm{c}$ oxidase (COX), the complex IV of the respiratory chain lower than in the corresponding control cybrid group $[51,52]$. COX activity reduction was associated with reduced ATP in the cell and increased ROS production. Finally, AD cybrids contained more intracellular APP products than the control cybrids [53-56].

\section{Is There a Role for the Mitochondrial DNA in AD?}

Even if cybrid studies have suggested mtDNA contribution to mitochondrial dysfunction in $\mathrm{AD}$, studies attempting to identify mtDNA mutations in the brains of $\mathrm{AD}$ patients had limited success. For example, Elson et al. [57] sequenced the complete coding regions of 145 autoptic AD brain samples and 128 controls. They observed that the overall numbers of mtDNA nucleotide substitutions were the same for the $\mathrm{AD}$ and for the control sequences [57]. So far, no causative mtDNA mutations have been linked to AD. Chagnon and coworkers [58] reported that haplogroup $\mathrm{T}$ is underrepresented in $\mathrm{AD}$ patients, while haplogroup $\mathrm{J}$ seems to be overrepresented. By studying a sample of Italian subjects, Carrieri et al. [59] showed that haplogroups $\mathrm{K}$ and $\mathrm{U}$ were present at a lower frequency in $\mathrm{AD}$ patients, who were apolipoprotein (Apo) E4 carriers than in noncarriers, while in controls there was no association between the E4 allele and mtDNA haplogroups. This evidence suggests that $\mathrm{K}$ and $\mathrm{U}$ may act by neutralising the effect of the major $\mathrm{AD}$ risk factor, the E4 allele [59]. Another report demonstrated that males classified as haplogroup $U$ had a significantly increased risk of $\mathrm{AD}$, while females had a significantly decreased risk with the same U haplogroup [60]. Two additional studies, including only neuropathologically proven cases of $\mathrm{AD}$ in European patients indicated that mtDNA haplogroups were not associated with $\mathrm{AD}$, either individually or by grouping together closely related haplogroups [57, 61]. Another study did not suggest an aetiological role of haplogroup-associated polymorphisms [62]. In conclusion, although the suggestions of a possible relation between haplogroups $\mathrm{K}$ and $\mathrm{U}$ and risk of $\mathrm{AD}$ in Caucasian population, this field of research is still to be fully explored. To date, mtDNA haplogroups do not seem to play a major role in $\mathrm{AD}$ pathogenesis [63]. 


\section{APP, $A \beta$, and Mitochondria}

The human APP gene, first identified in 1987, is located on chromosome 21. Several evidences suggest that mutations in the $A P P$ gene are correlated to FAD and a related condition, cerebral amyloid angiopathy $[15,64]$. The cleavage of APP produces two major forms of $\mathrm{A} \beta$ peptides, $\mathrm{A} \beta 40$ (the prevalent isoform), and the more pathogenic and less expressed A $\beta 42$. A number of functions have been attributed to APP, but its function is not fully understood to date.

$\mathrm{Li}$ et al. have shown a casual relationship between oxidative stress and AD plaque pathology using APP mutant $\mathrm{MnSOD+/-heterozygous} \mathrm{knockout} \mathrm{mice.} \mathrm{In} \mathrm{addition,} \mathrm{Espos-}$ ito and coworkers showed that partial reduction in the main mitochondrial superoxide scavenger Sod 2 accelerates the onset of hAPP/A $\beta$-dependent behavioural abnormalities and worsens a range of AD-related molecular and pathological alterations $[65,66]$.

However, others preclinical models with transfected cells and transgenic mice have shown that overexpression of APP produces mitochondrial dysfunction through the block of the mitochondrial protein import machinery [67]. Oxidative stress may facilitate the expression of a protein involved in the generation of $\mathrm{A} \beta$ and the beta-secretase (BACE 1) [68] and, moreover, may induces a pathogenic PS1 conformational change in neurons, increasing $A \beta 42 / 40$ ratio, as shown by in vitro models [69]. $\beta$-amyloid protein $(A \beta)$ interacts with the binding alcohol dehydrogenase protein (ABAD), a mitochondrial-matrix protein, causing mitochondrial oxidative damage and deficiency of the respiratory complexes [70, 71]. In view of the fact that $A \beta$ is not produced locally in the mitochondrion [67], Hansson Petersen and coworkers investigated the mechanisms by which $\mathrm{A} \beta$ is taken up by mitochondria [72]. The most important system providing the translocation of $\mathrm{A} \beta$ precursors with mitochondrial target signals involves both the translocase of the outer membrane (TOM) and the translocase of the inner membrane (TIM). Targeting signals are first recognized by TOM receptors (Tom20, Tom22, and Tom70), and then traslocated by Tom40, the general import pore of TOM [73, 74]. Subsequently, $\mathrm{A} \beta$ precursors are directed to the matrix via the Tim 23 complex [74]. In isolated rat mitochondria has been observed that $\mathrm{A} \beta$ is imported into mitochondria via the TOM complex [72]. With the preincubation of mitochondria with antibodies directed toward Tom20, Tom40, or Tom70, the import of $A \beta$ is decreased [72]. The import into mitochondria was insensitive to the mitochondrial membrane potential dissipater valinomycin, indicating that it is independent of the membrane potential of the mitochondrion [72]. $\mathrm{A} \beta$ also has many other functions such as interaction with HtrA2/Omi, a proapoptotic serine protease released into the cytoplasm by mitochondria on apoptotic stimulation [75], inhibition of ketoglutarate dehydrogenase complex, and reduction of COX activity [76]. An important role of $A \beta$ in modulating proteins involved in mitochondrial fission/fusion processes was recently suggested. Mitochondria are highly dynamic organelles, ranging from giant tubular networks to small round entities through rapid and reversible fission and fusion processes. In hippocampal tissues of AD patients, reduced levels GTPase dynamin-like protein 1 (DLP1), OPA1, Mfn1, and Mfn2 and increased levels of Fis1 have been found, suggesting impaired mitochondrial dynamics in favour of fission. A proper balance of fusion and fission proteins is fundamental for the correct distribution of the mitochondria in the cells [77]. This is particularly important for neurons that may have very long axons and also for the function of synapses, which are subcellular regions with high metabolic requirement [78].

\section{Tau Protein and Mitochondria}

Tau is a normal neuronal protein, which modulates microtubule-based functions. Multiple studies demonstrated that tau plays a crucial role in the pathogenesis of $\mathrm{AD}$, given that in animal models of $\mathrm{AD}$ reducing tau levels attenuates neuronal dysfunction $[79,80]$, and in humans the extent of tau pathology correlates with cognitive impairment [81]. Neurofibrillary tangles (NFTs), insoluble fibrillar intraneuronal accumulations of modified tau protein, are important and define hallmarks of the disease. The main modification of tau in the NFTs is hyperphosphorylation; however, numerous modifications have been reported, including truncation, acetylation, and nitration $[82,83]$. As mentioned above, reduced cell energy due to complexes I and IV inhibition promotes tau phosphorylation [84], suggesting that mitochondrial damage could play a role in facilitating tangles formation and thus neurodegeneration. The products of the toxic action of ROS may facilitate the self-assembly of the Tau protein into fibrillary polymers similar to the paired helical filaments present in the brain of AD patients [85]. On the other hand tau accumulation caused mitochondrial dysfunction in a mouse model of $\mathrm{AD}$ [86].

\section{Vascular Factors and Mitochondrial Dysfunction}

Pathological studies have shown reduced number of cerebral microvessels, decreased capillary diameters, and increased capillary densities in AD brains [87], with atrophy of smooth muscle cells in brain vessels and attenuation of capillary endothelium, resulting in the rupture of the vessel wall [88]. Atherosclerosis has also been observed in the circle of Willis and in large leptomeningeal vessels $[89,90]$.

Cerebral hypoperfusion may be a preclinical condition of $\mathrm{AD}$ [91]. The reduced cerebral blood flow (CBF) may play a critical role in the degenerative cascade leading to AD. Mitochondria in vulnerable cells show signs of damage during ischemia [92]. Chronic hypoperfusion can trigger mitochondrial dysfunction in vascular cells, which, in turn, will enhance both production and accumulation of ROS. When the ETC is inhibited, including when the regional CBF is reduced, the electrons are donated directly to oxygen to give an anion superoxide. Superoxide can react with nitric oxide (NO) to form peroxynitrite, which is severely damaging for DNA and RNA. NO, which acts as vasodilator, is reduced by increased levels of ROS. Thus, increased oxidative stress interferes with NO function and vascular relaxation. 
This scenario could play a role in the vascular abnormalities underlying metabolic defects in AD [92]. Moreover, increased levels of oxidative stress have been reported in AD brains [90]. Immunocytochemical studies revealed that the regional distribution of RNA oxidation in the brain was consistent with the selective neuronal vulnerability [80]. There were increased levels of 8-hydroxyguanosine (8-OHG) in the hippocampus and cerebral neocortex in $\mathrm{AD}$, whereas no alteration in the 8-OHG levels was found in the cerebellum [93]. Oxidized RNAs were localized predominantly in neuronal and endothelial cells compared with the glia [94], and oxidized RNA in endothelial cells could have a key role in the degenerative cascade [90]. Aliyev and coworkers [95] described the ultrastructural features of vascular and mitochondrial alterations in vascular wall cells from human $\mathrm{AD}$ brain biopsies. As expected, there was a higher degree of $\mathrm{A} \beta$ deposition in the vascular walls in $\mathrm{AD}$ compared to agematched controls [95]. Moreover, increased levels of 8-OHG and significantly more mitochondrial abnormalities were seen in the vascular endothelium and in the perivascular cells of the microvessels where atherosclerotic lesions occurred [96]. These features were absent in unaffected regions of $\mathrm{AD}$ or in age-matched control subjects [95], suggesting that vascular wall cells, and especially their mitochondria, may be a central target for oxidative damage before the development of AD pathology [90].

\section{Conclusion}

$\mathrm{AD}$ is the most common form of adult onset dementia. Its etiology is complex, and only a minority of cases are genetically inherited (FAD). The etiology of sporadic forms of $\mathrm{AD}(\mathrm{SAD})$ is still a controversial area. The relationship between mitochondrial pathology and APP and beta-amyloid metabolism is controversial as well. For instance, the cellular trafficking of APP and beta-amyloid are quite well understood but there is not a general consensus about their import in the mitochondria $[97,98]$. There is still a strong need for a better understanding of these connections.

Multiple interactions among genetic and environmental factors appear to be causative of the sporadic forms. Accumulation of somatic mtDNA mutations accelerates normal aging, leads to oxidative damage, and causes energy failure, increased production of ROS and amyloid accumulation, which in a vicious manner reinforces the mitochondrion damage, the impairment of the mitochondrial respiration, and the accumulation of ROS. Endothelial mtDNA deletions themselves seem to contribute to the pathogenesis, but the exact mechanism of that is still unclear.

Most likely, the mtDNA does not play a primary role, and, therefore, it could be involved subsequently [90]. A recent, large study excluded any association between $\mathrm{AD}$ and genetic variation of mtDNA, and meta-analysis of the available data showed no evidence of an association with $\mathrm{AD}$ [96]. It will be important to develop a better understanding of the role of oxidative stress and mitochondrial energy metabolism, and its links with the amyloid hypothesis and the endothelial and vascular dysfunction observed in AD [90], since it may lead to the development of more effective treatment strategies for this devastating disorder.

\section{Conflict of Interests}

The authors declare that there is no conflict of interests regarding the publication of this paper.

\section{References}

[1] A. Alzheimer, Über einen Eigenartigen Schweren Erkrankungsprozess der Hirnrinde, Neurologisches Centralblatt, Tübingen, Germany, 1906.

[2] A. Alzheimer, "Über eine eigenartige Erkrankung der Hirnrinde," Allgemeine Zeitschrift für Psychiatrie und PsychischGerichtliche Medizin, vol. 64, pp. 146-148, 1907.

[3] G. Perusini, "Über klinisch und histologisch eigenartige psychische Erkrankungen des späteren Lebensalters," Histologische und Histopathologlsche Arbeiten, vol. 3, no. 2, pp. 197-351, 1910.

[4] G. B. Irvine, O. M. El-Agnaf, G. M. Shankar, and D. M. Walsh, "Protein aggregation in the brain: the molecular basis for Alzheimer's and Parkinson's diseases," Molecular Medicine, vol. 14, no. 7-8, pp. 451-464, 2008.

[5] M. Mancuso, D. Orsucci, A. LoGerfo, V. Calsolaro, and G. Siciliano, "Clinical features and pathogenesis of Alzheimer's disease: involvement of mitochondria and mitochondrial DNA," Advances in Experimental Medicine and Biology, vol. 685, pp. 34-44, 2010.

[6] R. Katzman, "Alzheimer's disease," The New England Journal of Medicine, vol. 314, no. 15, pp. 964-973, 1986.

[7] M. A. Smith, "Alzheimer disease," International Review of Neurobiology, vol. 42, pp. 1-54, 1998.

[8] C. L. Masters, G. Simms, N. A. Weinman, G. Multhaup, B. L. McDonald, and K. Beyreuther, "Amyloid plaque core protein in Alzheimer disease and Down syndrome," Proceedings of the National Academy of Sciences of the United States of America, vol. 82, no. 12, pp. 4245-4249, 1985.

[9] I. Grundke-Iqbal, K. Iqbal, M. Quinlan, Y. C. Tung, M. S. Zaidi, and H. M. Wisniewski, "Microtubule associated protein tau. A component of Alzheimer paired helical filaments," The Journal of Biological Chemistry, vol. 261, no. 13, pp. 6084-6089, 1986.

[10] W. E. Klunk, H. Engler, A. Nordberg et al., "Imaging brain amyloid in Alzheimer's disease with Pittsburgh Compound-B," Annals of Neurology, vol. 55, no. 3, pp. 306-319, 2004.

[11] M. T. Fodero-Tavoletti, N. Okamura, S. Furumoto et al., “ ${ }_{18} \mathrm{~F}-$ THK523: a novel in vivo tau imaging ligand for Alzheimer's disease," Brain, vol. 134, no. 4, pp. 1089-1100, 2011.

[12] R. C. Petersen, R. Doody, A. Kurz et al., "Current concepts in mild cognitive impairment," Archives of Neurology, vol. 58, no. 12, pp. 1985-1992, 2001.

[13] D. S. Knopman, B. F. Boeve, and R. C. Petersen, "Essentials of the proper diagnoses of mild cognitive impairment, dementia, and major subtypes of dementia," Mayo Clinic Proceedings, vol. 78, no. 10, pp. 1290-1308, 2003.

[14] R. C. Petersen, "Mild cognitive impairment as a diagnostic entity," Journal of Internal Medicine, vol. 256, no. 3, pp. 183-194, 2004.

[15] B. Nacmias, I. Piaceri, S. Bagnoli, A. Tedde, S. Piacentini, and S. Sorbi, "Genetics of Alzheimer's disease and frontotemporal dementia," Current Molecular Medicine, vol. 14, no. 8, pp. 9931000, 2014. 
[16] J. Hardy and D. J. Selkoe, “The amyloid hypothesis of Alzheimer's disease: progress and problems on the road to therapeutics," Science, vol. 297, no. 5580, pp. 353-356, 2002.

[17] R. Vassar, B. D. Bennett, S. Babu-Khan et al., " $\beta$-secretase cleavage of Alzheimer's amyloid precursor protein by the transmembrane aspartic protease BACE," Science, vol. 286, no. 5440, pp. 735-741, 1999.

[18] W. T. Kimberly, M. J. LaVoie, B. L. Ostaszewski, W. Ye, M. S. Wolfe, and D. J. Selkoe, " $\gamma$-Secretase is a membrane protein complex comprised of presenilin, nicastrin, aph-1, and pen-2," Proceedings of the National Academy of Sciences of the United States of America, vol. 100, no. 11, pp. 6382-6387, 2003.

[19] D. M. Walsh, B. P. Tseng, R. E. Rydel, M. B. Podlisny, and D. J. Selkoe, "The oligomerization of amyloid beta-protein begins intracellularly in cells derived from human brain," Biochemistry, vol. 39, no. 35, pp. 10831-10839, 2000.

[20] R. H. Takahashi, C. G. Almeida, P. F. Kearney et al., "Oligomerization of Alzheimers beta-amyloid within processes and synapses of cultured neurons and brain," The Journal of Neuroscience, vol. 24, no. 14, pp. 3592-3599, 2004.

[21] G. K. Gouras, J. Tsai, J. Naslund et al., "Intraneuronal A $\beta 42$ accumulation in human brain," The American Journal of Pathology, vol. 156, no. 1, pp. 15-20, 2000.

[22] O. Wirths, G. Multhaup, and T. A. Bayer, "A modified $\beta$ amyloid hypothesis: intraneuronal accumulation of the $\beta$ amyloid peptide-the first step of a fatal cascade," Journal of Neurochemistry, vol. 91, no. 3, pp. 513-520, 2004.

[23] C. S. Casley, L. Canevari, J. M. Land, J. B. Clark, and M. A. Sharpe, "Beta amyloid inhibts integrated mitochondrial respiration and key enzyme activities," Journal of Neurochemistry, vol. 80, no. 1, pp. 91-100, 2002.

[24] S. Raha and B. H. Robinson, "Mitochondria, oxygen free radicals, and apoptosis," American Journal of Medical Genetics, vol. 106, no. 1, pp. 62-70, 2001.

[25] D. J. Bonda, X. Wang, G. Perry et al., "Oxidative stress in Alzheimer disease: a possibility for prevention," Neuropharmacology, vol. 59, no. 4-5, pp. 290-294, 2010.

[26] E. C. Ienco, C. Simoncini, D. Orsucci et al., "May 'mitochondrial eve' and mitochondrial haplogroups play a role in neurodegeneration and alzheimer's disease?" International Journal of Alzheimer's Disease, vol. 2011, Article ID 709061, 11 pages, 2011.

[27] N. P. Azari, K. D. Pettigrew, M. B. Schapiro et al., "Early detection of Alzheimer's disease: a statistical approach using positron emission tomographic data," Journal of Cerebral Blood Flow \& Metabolism, vol. 13, no. 3, pp. 438-447, 1993.

[28] J. P. Blass, R. K.-F. Sheu, and G. E. Gibson, "Inherent abnormalities in energy metabolism in Alzheimer disease: interaction with cerebrovascular compromise," Annals of the New York Academy of Sciences, vol. 903, pp. 204-221, 2000.

[29] D. C. Wallace, "Mitochondrial diseases in man and mouse," Science, vol. 283, no. 5407, pp. 1482-1488, 1999.

[30] G. E. Gibson, V. Haroutunian, H. Zhang et al., "Mitochondrial damage in Alzheimer's disease varies with apolipoprotein E genotype," Annals of Neurology, vol. 48, no. 3, pp. 297-303, 2000.

[31] P. Bubber, V. Haroutunian, G. Fisch, J. P. Blass, and G. E. Gibson, "Mitochondrial abnormalities in Alzheimer brain: mechanistic implications," Annals of Neurology, vol. 57, no. 5, pp. 695-703, 2005.

[32] W. S. Liang, E. M. Reiman, J. Valla et al., "Alzheimer's disease is associated with reduced expression of energy metabolism genes in posterior cingulate neurons," Proceedings of the National
Academy of Sciences of the United States of America, vol. 105, no. 11, pp. 4441-4446, 2008.

[33] K. Henze and W. Martin, "Evolutionary biology: essence of mitochondria," Nature, vol. 426, no. 6963, pp. 127-128, 2003.

[34] S. Di Mauro and E. A. Schon, "Mitochondrial respiratory-chain diseases," The New England Journal of Medicine, vol. 348, no. 26, pp. 2656-2668, 2003.

[35] H. Noji and M. Yoshida, "The rotary machine in the cell, ATP synthase," The Journal of Biological Chemistry, vol. 276, no. 3, pp. 1665-1668, 2001.

[36] M. F. Beal, "Mitochondria take center stage in aging and neurodegeneration," Annals of Neurology, vol. 58, no. 4, pp. 495505, 2005.

[37] J. B. Schulz, J. Lindenau, J. Seyfried, and J. Dichgans, "Glutathione, oxidative stress and neurodegeneration," European Journal of Biochemistry, vol. 267, no. 16, pp. 4904-4911, 2000.

[38] E. Mariani, M. C. Polidori, A. Cherubini, and P. Mecocci, "Oxidative stress in brain aging, neurodegenerative and vascular diseases: an overview," Journal of Chromatography B: Analytical Technologies in the Biomedical and Life Sciences, vol. 827, no. 1, pp. 65-75, 2005.

[39] W. J. H. Koopman, F. Distelmaier, J. A. M. Smeitink, and P. H. G. M. Willems, "OXPHOS mutations and neurodegeneration," The EMBO Journal, vol. 32, no. 1, pp. 9-29, 2013.

[40] J. Avila, "Common mechanisms in neurodegeneration," Nature Medicine, vol. 16, no. 12, article 1372, 2010.

[41] M. Filosto, M. Scarpelli, M. S. Cotelli et al., "The role of mitochondria in neurodegenerative diseases," Journal of Neurology, vol. 258, no. 10, pp. 1763-1774, 2011.

[42] X. Zhu, G. Perry, M. A. Smitha, and X. Wang, "Abnormal mitochondrial dynamics in the pathogenesis of Alzheimer's disease," Journal of Alzheimer's Disease, vol. 33, supplement 1, pp. S253-S262, 2013.

[43] P. I. Moreira, C. Carvalho, X. Zhu, M. A. Smith, and G. Perry, "Mitochondrial dysfunction is a trigger of Alzheimer's disease pathophysiology," Biochimica et Biophysica Acta, vol. 1802, no. 1, pp. 2-10, 2010.

[44] D. B. Zorov, M. Juhaszova, and S. J. Sollott, "Mitochondrial reactive oxygen species (ROS) and ROS-induced ROS release," Physiological Reviews, vol. 94, no. 3, pp. 909-950, 2014.

[45] M. Mancuso, F. Coppedè, L. Murri, and G. Siciliano, "Mitochondrial cascade hypothesis of Alzheimer's disease: myth or reality?" Antioxidants and Redox Signaling, vol. 10, pp. 16311646, 2007.

[46] R. X. Santos, S. C. Correia, X. Wang et al., "A synergistic dysfunction of mitochondrial fission/fusion dynamics and mitophagy in Alzheimer's disease," Journal of Alzheimer's Disease, vol. 20, no. 2, pp. S401-S412, 2010.

[47] M. Sano, C. Ernesto, R. G. Thomas et al., "A controlled trial of selegiline, alpha-tocopherol, or both as treatment for Alzheimer's disease. The Alzheimer's Disease Cooperative Study," The New England Journal of Medicine, vol. 336, no. 17, pp. 1216-1222, 1997.

[48] R. C. Petersen, R. G. Thomas, M. Grundman et al., "Vitamin E and donepezil for the treatment of mild cognitive impairment," The New England Journal of Medicine, vol. 352, no. 23, pp. 23792388, 2005.

[49] M. Dumont, K. Kipiani, F. Yu et al., “Coenzyme Q10 decreases amyloid pathology and improves behavior in a transgenic mouse model of Alzheimer's disease," Journal of Alzheimer's Disease, vol. 27, no. 1, pp. 211-223, 2011. 
[50] R. H. Swerdlow, J. K. Parks, D. S. Cassarino et al., "Cybrids in Alzheimer's disease: a cellular model of the disease?" Neurology, vol. 49, no. 4, pp. 918-925, 1997.

[51] G. N. Bijur, R. E. Davis, and R. S. Jope, "Rapid activation of heat shock factor-1 DNA binding by $\mathrm{H}_{2} \mathrm{O}_{2}$ and modulation by glutathione in human neuroblastoma and Alzheimer's disease cybrid cells," Molecular Brain Research, vol. 71, no. 1, pp. 69-77, 1999.

[52] S. M. Cardoso, I. Santana, R. H. Swerdlow, and C. R. Oliveira, "Mitochondria dysfunction of Alzheimer's disease cybrids enhances A $\beta$ toxicity," Journal of Neurochemistry, vol. 89, no. 6, pp. 1417-1426, 2004.

[53] P. de Sarno, G. N. Bijur, R. Lu, R. E. Davis, and R. S. Jope, "Alterations in muscarinic receptor-coupled phosphoinositide hydrolysis and AP-1 activation in Alzheimer's disease cybrid cells," Neurobiology of Aging, vol. 21, no. 1, pp. 31-38, 2000.

[54] I. G. Onyango, J.-Y. Ahn, J. B. Tuttle, J. P. Bennett Jr., and R. H. Swerdlow, "Nerve growth factor attenuates oxidant-induced beta-amyloid neurotoxicity in sporadic Alzheimer's disease cybrids," Journal of Neurochemistry, vol. 114, no. 6, pp. 16051618, 2010.

[55] H. Zhang, Y. Liu, M. Lao, Z. Ma, and X. Yi, "Puerarin protects Alzheimer's disease neuronal cybrids from oxidant-stress induced apoptosis by inhibiting pro-death signaling pathways," Experimental Gerontology, vol. 46, no. 1, pp. 30-37, 2011.

[56] L. Chen, S.-E. Yoo, R. Na, Y. Liu, and Q. Ran, "Cognitive impairment and increased $\mathrm{A} \beta$ levels induced by paraquat exposure are attenuated by enhanced removal of mitochondrial $\mathrm{H}_{2} \mathrm{O}_{2}$," Neurobiology of Aging, vol. 33, no. 2, pp. 432.e15-432.e26, 2012.

[57] J. L. Elson, C. Herrnstadt, G. Preston et al., "Does the mitochondrial genome play a role in the etiology of Alzheimer's disease?" Human Genetics, vol. 119, no. 3, pp. 241-254, 2006.

[58] P. Chagnon, M. Gee, M. Filion, Y. Robitaille, M. Belouchi, and D. Gauvreau, "Phylogenetic analysis of the mitochondrial genome indicates significant differences between patients with Alzheimer disease and controls in a French-Canadian founder population," American Journal of Medical Genetics, vol. 85, no. 1, pp. 20-30, 1999.

[59] G. Carrieri, M. Bonafè, M. de Luca et al., "Mitochondrial DNA haplogroups and APOE4 allele are non-independent variables in sporadic Alzheimer's disease," Human Genetics, vol. 108, no. 3, pp. 194-198, 2001.

[60] J. M. van der Walt, Y. A. Dementieva, E. R. Martin et al., "Analysis of European mitochondrial haplogroups with Alzheimer disease risk," Neuroscience Letters, vol. 365, no. 1, pp. 28-32, 2004.

[61] P. F. Chinnery, G. A. Taylor, N. Howell et al., "Mitochondrial DNA haplogroups and susceptibility to $\mathrm{AD}$ and dementia with Lewy bodies," Neurology, vol. 55, no. 2, pp. 302-304, 2000.

[62] M. Mancuso, M. Nardini, D. Micheli et al., "Lack of association between mtDNA haplogroups and Alzheimer's disease in Tuscany," Neurological Sciences, vol. 28, no. 3, pp. 142-147, 2007.

[63] M. Mancuso, M. Filosto, D. Orsucci, and G. Siciliano, "Mitochondrial DNA sequence variation and neurodegeneration," Human Genomics, vol. 3, no. 1, pp. 71-78, 2008.

[64] E. Levy and M. D. Carman, "Mutation of the Alzheimer's disease amyloid gene in hereditary cerebral hemorrhage Dutch type," Science, vol. 248, no. 4959, pp. 1124-1126, 1990.

[65] F. Li, N. Y. Calingasan, F. Yu et al., "Increased plaque burden in brains of APP mutant MnSOD heterozygous knockout mice," Journal of Neurochemistry, vol. 89, no. 5, pp. 1308-1312, 2004.
[66] L. Esposito, J. Raber, L. Kekonius et al., "Reduction in mitochondrial superoxide dismutase modulates Alzheimer's disease-like pathology and accelerates the onset of behavioral changes in human amyloid precursor protein transgenic mice," The Journal of Neuroscience, vol. 26, no. 19, pp. 5167-5179, 2006.

[67] H. K. Anandatheerthavarada, G. Biswas, M.-A. Robin, and N. G. Avadhani, "Mitochondrial targeting and a novel transmembrane arrest of Alzheimer's amyloid precursor protein impairs mitochondrial function in neuronal cells," Journal of Cell Biology, vol. 161, no. 1, pp. 41-54, 2003.

[68] K. Xiong, H. Cai, X.-G. Luo, R. G. Struble, R. W. Clough, and $\mathrm{X}$.-X. Yan, "Mitochondrial respiratory inhibition and oxidative stress elevate $\beta$-secretase (BACE1) proteins and activity in vivo in the rat retina," Experimental Brain Research, vol. 181, no. 3, pp. 435-446, 2007.

[69] L. Wahlster, M. Arimon, N. Nasser-Ghodsi et al., "Presenilin1 adopts pathogenic conformation in normal aging and in sporadic Alzheimer's disease," Acta Neuropathologica, vol. 125, no. 2, pp. 187-199, 2013.

[70] E. Borger, L. Aitken, K. E. A. Muirhead et al., "Mitochondrial $\beta$-amyloid in Alzheimer's disease," Biochemical Society Transactions, vol. 39, no. 4, pp. 868-873, 2011.

[71] E. Borger, L. Aitken, H. Du, W. Zhang, F. J. Gunn-Moore, and S. S. D. Yan, "Is amyloid binding alcohol dehydrogenase a drug target for treating Alzheimer's disease?" Current Alzheimer Research, vol. 10, no. 1, pp. 21-29, 2013.

[72] C. A. Hansson Petersen, N. Alikhani, H. Behbahani et al., "The amyloid $\beta$-peptide is imported into mitochondria via the TOM import machinery and localized to mitochondrial cristae," Proceedings of the National Academy of Sciences of the United States of America, vol. 105, no. 35, pp. 13145-13150, 2008.

[73] W. Neupert and J. M. Herrmann, "Translocation of proteins into mitochondria," Annual Review of Biochemistry, vol. 76, pp. 723749, 2007.

[74] P. Rehling, N. Pfanner, and C. Meisinger, "Insertion of hydrophobic membrane proteins into the inner mitochondrial membrane-a guided tour," Journal of Molecular Biology, vol. 326, no. 3, pp. 639-657, 2003.

[75] D. Lindholm, O. Eriksson, and L. Korhonen, "Mitochondrial proteins in neuronal degeneration," Biochemical and Biophysical Research Communications, vol. 321, no. 4, pp. 753-758, 2004.

[76] C. S. Casley, L. Canevari, J. M. Land, J. B. Clark, and M. A. Sharpe, "Beta-amyloid inhibits integrated mitochondrial respiration and key enzyme activities," Journal of Neurochemistry, vol. 80, no. 1, pp. 91-100, 2002.

[77] S. A. Detmer and D. C. Chan, "Functions and dysfunctions of mitochondrial dynamics," Nature Reviews Molecular Cell Biology, vol. 8, no. 11, pp. 870-879, 2007.

[78] A. E. Frazier, C. Kiu, D. Stojanovski, N. J. Hoogenraad, and M. T. Ryan, "Mitochondrial morphology and distribution in mammalian cells," Biological Chemistry, vol. 387, no. 12, pp. 15511558, 2006.

[79] L. M. Ittner, Y. D. Ke, F. Delerue et al., "Dendritic function of tau mediates amyloid- $\beta$ toxicity in Alzheimer's disease mouse models," Cell, vol. 142, no. 3, pp. 387-397, 2010.

[80] E. D. Roberson, B. Halabisky, J. W. Yoo et al., "Amyloid- $\beta /$ fyninduced synaptic, network, and cognitive impairments depend on tau levels in multiple mouse models of alzheimer's disease," The Journal of Neuroscience, vol. 31, no. 2, pp. 700-711, 2011.

[81] A. L. Guillozet, S. Weintraub, D. C. Mash, and M. Marsel Mesulam, "Neurofibrillary tangles, amyloid, and memory in 
aging and mild cognitive impairment," Archives of Neurology, vol. 60, no. 5, pp. 729-736, 2003.

[82] C. S. Arnold, G. V. W. Johnson, R. N. Cole, D. L.-Y. Dong, M. Lee, and G. W. Hart, "The microtubule-associated protein tau is extensively modified with $O$-linked $N$-acetylglucosamine," The Journal of Biological Chemistry, vol. 271, no. 46, pp. 28741-28744, 1996.

[83] T. J. Cohen, J. L. Guo, D. E. Hurtado et al., "The acetylation of tau inhibits its function and promotes pathological tau aggregation," Nature Communications, vol. 2, no. 1, article 252, 2011.

[84] S. Melov, P. A. Adlard, K. Morten et al., "Mitochondrial oxidative stress causes hyperphosphorylation of tau," PLoS ONE, vol. 2, no. 6, article e536, 2007.

[85] I. Santa-María, F. Hernández, C. Pérez Martín, J. Avila, and F. J. Moreno, "Quinones facilitate the self-assembly of the phosphorylated tubulin binding region of tau into fibrillar polymers," Biochemistry, vol. 43, no. 10, pp. 2888-2897, 2004.

[86] K. J. Kopeikina, G. A. Carlson, R. Pitstick et al., "Tau accumulation causes mitochondrial distribution deficits in neurons in a mouse model of tauopathy and in human Alzheimer's disease brain," The American Journal of Pathology, vol. 179, no. 4, pp. 2071-2082, 2011.

[87] G. L. Mancardi, F. Perdelli, C. Rivano, A. Leonardi, and O. Bugiani, "Thickening of the basement membrane of cortical capillaries in Alzheimer's disease," Acta Neuropathologica, vol. 49, no. 1, pp. 79-83, 1980.

[88] G. Perry, M. A. Smith, C. E. McCann, S. L. Siedlak, P. K. Jones, and R. P. Friedland, "Cerebrovascular muscle atrophy is a feature of Alzheimer's disease," Brain Research, vol. 791, no. 1-2, pp. 63-66, 1998.

[89] A. E. Roher, C. Esh, A. Rahman, T. A. Kokjohn, and T. G. Beach, "Atherosclerosis of cerebral arteries in Alzheimer disease," Stroke, vol. 35, no. 11, pp. 2623-2627, 2004.

[90] D. Orsucci, M. Mancuso, E. Caldarazzo Ienco, C. Simoncini, G. Siciliano, and U. Bonuccelli, "Vascular factors and mitochondrial dysfunction: a central role in the pathogenesis of Alzheimer's disease," Current Neurovascular Research, vol. 10, no. 1, pp. 76-80, 2013.

[91] A. Ruitenberg, T. den Heijer, S. L. M. Bakker et al., "Cerebral hypoperfusion and clinical onset of dementia: the Rotterdam Study," Annals of Neurology, vol. 57, no. 6, pp. 789-794, 2005.

[92] X. Zhu, M. A. Smith, K. Honda et al., "Vascular oxidative stress in Alzheimer disease," Journal of the Neurological Sciences, vol. 257, no. 1-2, pp. 240-246, 2007.

[93] A. Nunomura, G. Perry, M. A. Pappolla et al., "RNA oxidation is a prominent feature of vulnerable neurons in Alzheimer's disease," The Journal of Neuroscience, vol. 19, no. 6, pp. 19591964, 1999.

[94] A. Nunomura, S. Chiba, C. F. Lippa et al., "Neuronal RNA oxidation is a prominent feature of familial Alzheimer's disease," Neurobiology of Disease, vol. 17, no. 1, pp. 108-113, 2004.

[95] A. Aliyev, S. G. Chen, D. Seyidova et al., "Mitochondria DNA deletions in atherosclerotic hypoperfused brain microvessels as a primary target for the development of Alzheimer's disease," Journal of the Neurological Sciences, vol. 229-230, pp. 285-292, 2005.

[96] G. Hudson, R. Sims, D. Harold et al., "No consistent evidence for association between mtDNA variants and Alzheimer disease," Neurology, vol. 78, no. 14, pp. 1038-1042, 2012.

[97] L. Rajendran and W. Annaert, "Membrane trafficking pathways in Alzheimer's disease," Traffic, vol. 13, no. 6, pp. 759-770, 2012.
[98] C. Haass, C. Kaether, G. Thinakaran, and S. Sisodia, “Trafficking and proteolytic processing of APP," Cold Spring Harbor Perspectives in Medicine, vol. 2, no. 5, Article ID a006270, 2012. 


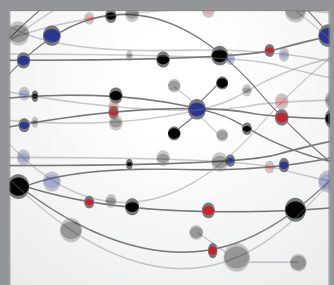

The Scientific World Journal
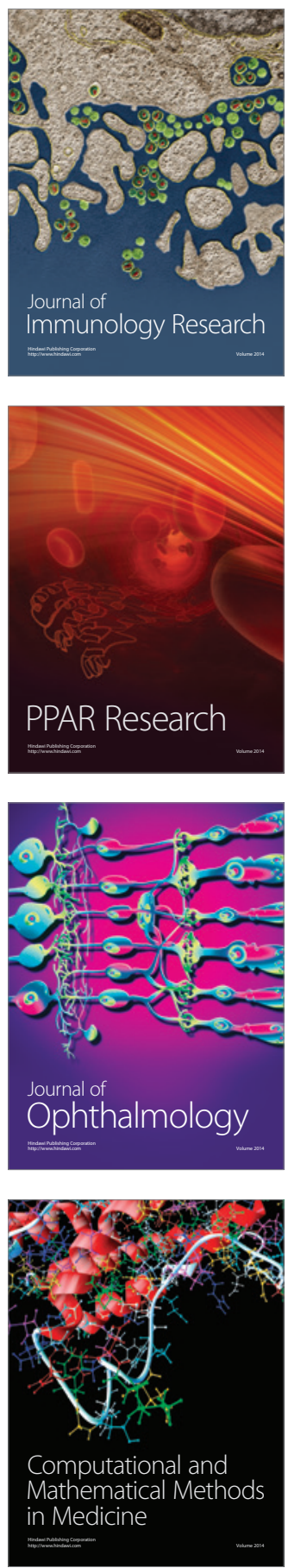

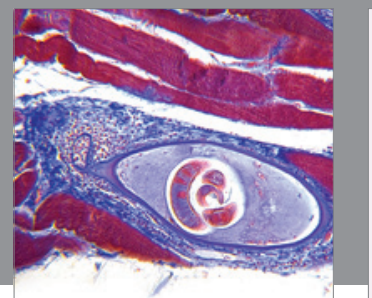

Gastroenterology

Research and Practice
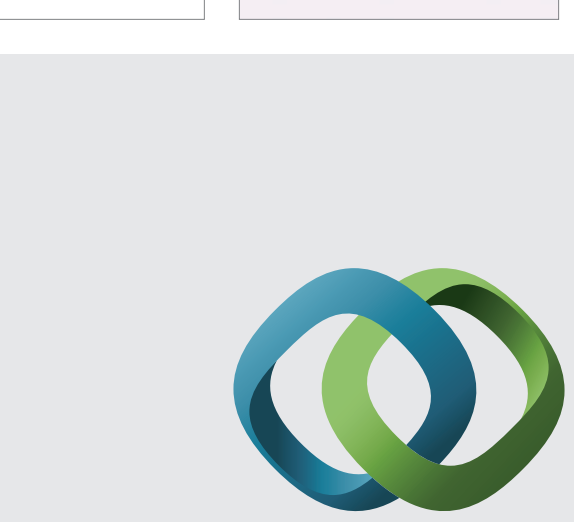

\section{Hindawi}

Submit your manuscripts at

http://www.hindawi.com
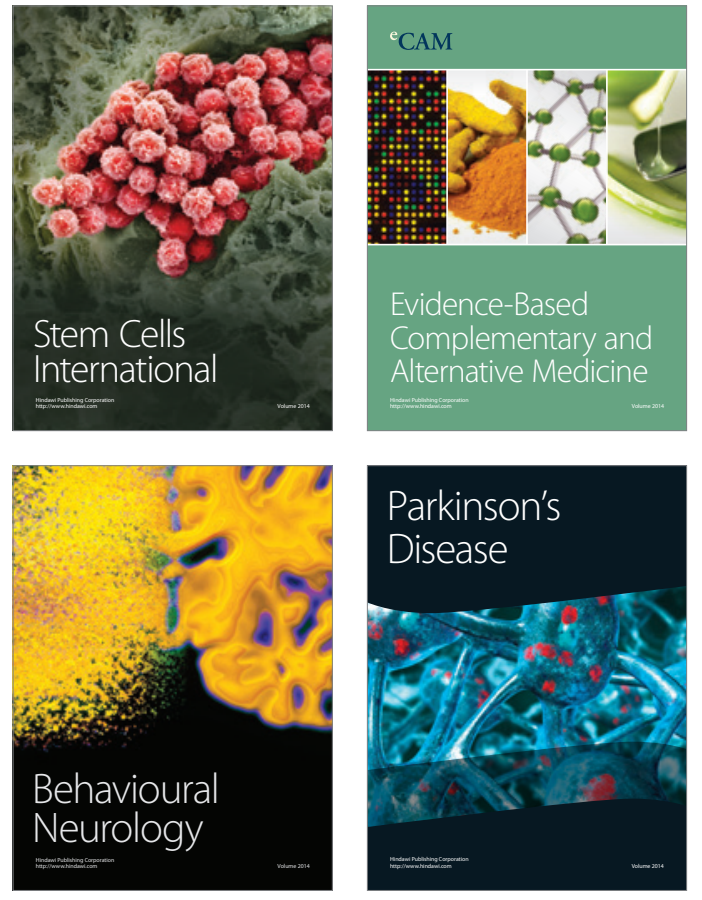
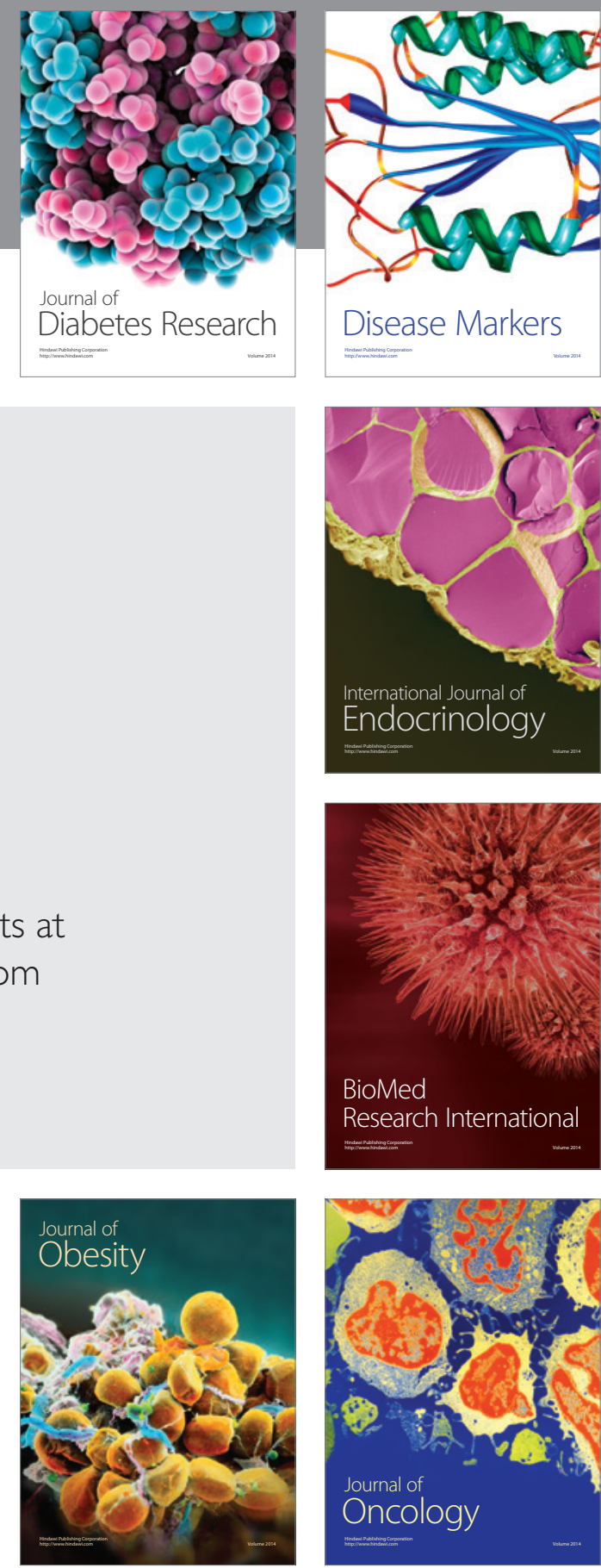

Disease Markers
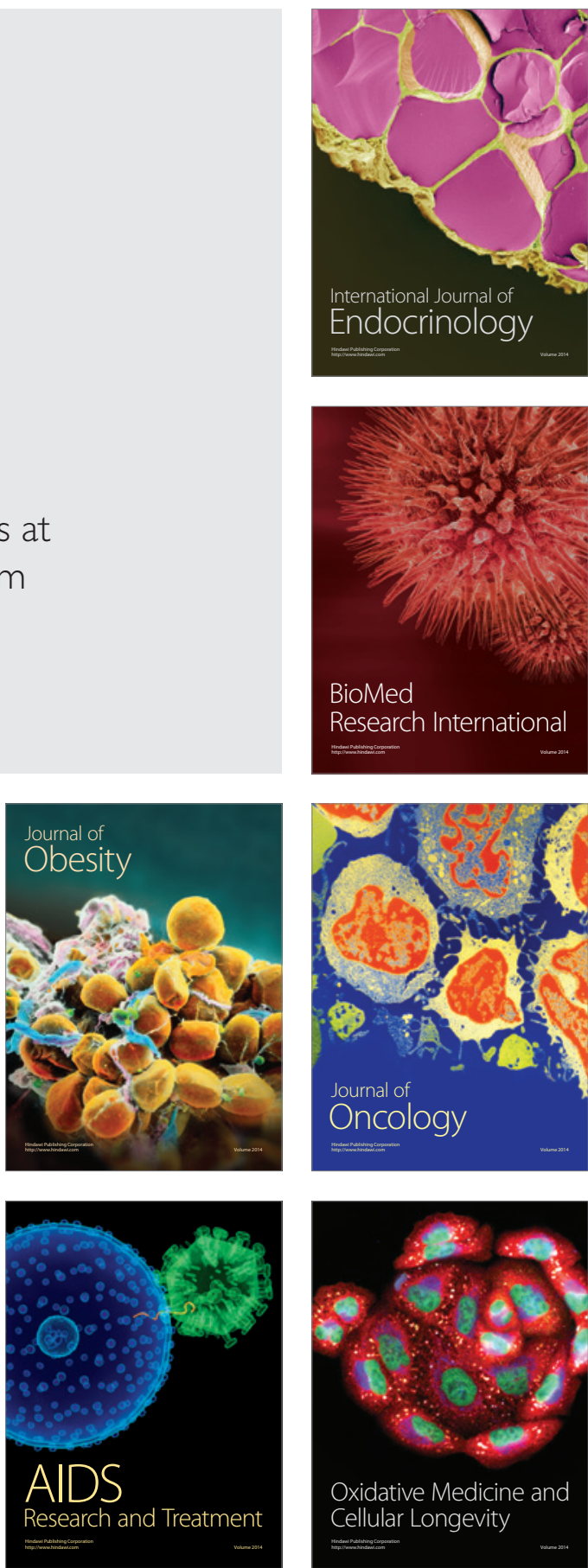